Tropical Journal of Pharmaceutical Research March 2017; 16 (3): 673-679

ISSN: $1596-5996$ (print); 1596-9827 (electronic)

(C) Pharmacotherapy Group, Faculty of Pharmacy, University of Benin, Benin City, 300001 Nigeria.

All rights reserved.

Available online at http://www.tjpr.org

Original Research Article

http://dx.doi.org/10.4314/tjpr.v16i3.24

\title{
Epileptic seizures in patients with glioma: A single centre- based study in China
}

\author{
Song Huang ${ }^{1,2 \star}$, Junli $\mathrm{CaO}^{3}$ and Lei Wang ${ }^{2}$ \\ ${ }^{1}$ Department of Neurosurgery, Institute of Neurology, China Three Gorges University, ${ }^{2}$ Department of Neurosurgery, \\ ${ }^{3}$ Department of Radiology, Yichang Central People's Hospital, Yichang, Hubei 443003, China \\ ${ }^{*}$ For correspondence: Email: songhuang147@hotmail.com; Tel/Fax: 0086-717-6496666
}

Received: 19 October 2016

Revised accepted: 18 December 2016

\begin{abstract}
Purpose: To elucidate the outcomes of treatment and epidemiology of epilepsy related to glioma in a single center in Chinese patients.

Methods: Prescription medicines usage and clinical data were collected from medical records of 119 patients with gliomas between August 2009 and September 2015. Fisher's exact and Chi square tests were used for analysis of seizure incidence differences as per WHO Grades, histology, location as well as tumour number, age and sex.

Results: Preoperative seizures were noted in $33.8 \%$ of glioma patients. After surgery, all the patients were given antiepileptic drugs (AEDs) prophylactically. Ten patients (8.4\%) developed seizures in the initial postoperative week, and $73(61.3 \%)$ patients at the last follow-up period. Overall, seizure incidence was $74.2 \%$ in WHO Grade II, $68.4 \%$ in Grade III, and $56.6 \%$ in Grade IV glioma patients. Good seizure control and tolerance was demonstrated by levetiracetam. Phenytoin/carbamazepine were poorly tolerated due to adverse events. AED discontinuation was done in 54 patients and 14 patients developed seizures within 6 months and again needed AED.

Conclusion: Effective prophylaxis of seizure and acceptable adverse event profile are to be considered significantly in AEDs selection. Lower cognitive impairment risk and renal excretion associated with newer AEDs make them better than older therapeutic agents for epilepsy control in brain tumour patients.
\end{abstract}

Keywords: Brain tumour, Epilepsy, Glioma, Seizures, Levetiracetam, Phenytoin, Carbamazepine, Epidemiology

Tropical Journal of Pharmaceutical Research is indexed by Science Citation Index (SciSearch), Scopus, International Pharmaceutical Abstract, Chemical Abstracts, Embase, Index Copernicus, EBSCO, African Index Medicus, JournalSeek, Journal Citation Reports/Science Edition, Directory of Open Access Journals (DOAJ), African Journal Online, Bioline International, Open-J-Gate and Pharmacy Abstracts

\section{INTRODUCTION}

Epilepsy is typical in brain tumour patients, and its control plays imperative part in clinical management. Convulsions add up considerable morbidity in brain tumour patients [1]. Epilepsy in brain tumour patients are mostly symptomatic and localization associated, showing as either simple or complex seizures with/without secondary generalization.
A variety of factors influence epileptogenesis in brain tumour patients, namely tumour location and type, peritumoural environmental changes, and genetic factors. Hurdles of epilepsy medical treatment in brain tumour patients encompass the epilepsy refractoriness, which may be due to potential interactions of antiepileptic drugs (AEDs) as well as chemotherapeutic agents; multidrug-resistance proteins; and potential cognitive side-effects on utilization of AED besides prior damage to brain from radiotherapy or surgery $[2,3]$. 
The brain tumour incidence in epilepsy patients is around $4 \%$. The seizure frequency is around $30 \%$ or more in brain tumour patients, relying upon tumour type. Epilepsy is the major medical sign of tumour in nearly $30-50 \%$ of brain tumour patients; $10-30 \%$ of patients develop epilepsy during the tumour course [2-6].

There is a dearth of studies concerning seizures related to glioma. Our study objective was to elucidate outcomes of treatment and epidemiology of epilepsy related to glioma in a single center.

\section{METHODS}

This single center retrospective study was conducted at Department of Neurosurgery at Yichang Central People's hospital, China over the period, August 2009 to September 2015.

One hundred and nineteen successive patients having supratentorial gliomas in the Neurological Surgery Division of the hospital, in China, between August 2009 and September 2015 were included. Our study included 46 female and 73 male patients (average age: 57 years). All of the cases had pathologically confirmed diagnosis. Altogether, 98 astrocytic plus 21 oligodendroglial tumours were present. The summary of patient characteristics were mentioned in Table 1. Since our center has no standard guidelines set for AED utilization, the particular AED used, relied upon preference of clinicians. Prophylactic AED treatment was customarily initiated prior to surgery. Phenytoin was typically chosen for the seizure prophylaxis during surgery. To accomplish stable concentration in serum, phenytoin was initiated 2 or 3 days prior to surgery and proceeded for 6 days post-surgery. In cases of adverse reactions to phenytoin and inadequately controlled seizures by phenytoin, patients were prescribed phenobarbital via intramuscular injection or suppository during surgery.

Post-surgery, all of the patients with WHO Grade III or Grade IV tumours were on treatment with irradiation along with temozolomide adjuvant concurrently, as per Stupp protocol [7], while patients having Grade II tumours were on simple observation. Our study received Ethics Committee approval and confidentiality of patients was strictly maintained. Data were collected from medical records. The clinical as well as demographics variables obtained were noted below: age, sex, histology, seizure onset dates, AED usage, tumour location and seizure control etc.

\section{Data analysis}

Values are expressed as numbers and percentage. Fisher's exact and Chi square tests were used for analysis of difference in seizure incidence as per WHO Grades, histology, location as well as tumour number, age, and sex. Analysis of collected data was performed using SPSS v16.0 (SPSS Inc, Chicago, USA). $P \leq 0.05$ was regarded as statistically significant.

\section{RESULTS}

Overall, 83 patients died by the time of final followup. Post-surgery median survival time for all of the patients was 20.2 months. The median follow up duration was noted to be 39.6 months (ranging from $3.7-84.2$ months) for 36 surviving patients.

\section{Preoperative seizure incidence}

In $29(24.4 \%)$ patients, the presenting symptom was seizure. No significant statistical difference in the seizure incidence as primary symptom, among patients having oligodendroglia tumours $(40.9 \%)$ vs. astrocytic tumours $(22.7 \%) ;(p=$ $0.145)$ was noted. Seizure incidence in patients, as per WHO grade were as follows: Grade II tumours $47.2 \%$; Grade III tumours $28.4 \%$; and Grade IV tumours $19.6 \%$. The seizure incidence was significantly higher in patients having Grade II tumours than patients having Grade IV tumours $(p=0.026)$. Seizures were seen most commonly in younger $(<50 \mathrm{yrs}, 54.1 \%)$ than older patients $(\geq 50 \mathrm{yr}, p<0.001)$. Non-significant inclination for frontal lobe tumours to exhibit more frequent onset of seizures than the tumours present in other regions $(p=0.095)$. The clinical/ demographic characteristics are presented in Table 1. The seizure incidence as primary symptom and pertinent characteristics of patients are presented in Table 2.

Besides 29 patients, whose presenting symptom was seizures, additional 10 patients (8.4\%) developed seizures in between their first visit and surgery. AED was given quickly post one seizure had happened in all the patients having seizures. Phenytoin was the widely utilized AED (48.2\%), however physicians likewise as often as possible chose valproic acid $(24.1 \%)$ and phenobarbital (20.7\%).

Out of 80 patients with no seizures, 49 patients were given AED prophylactically prior to surgery. Phenytoin was the most widely utilized AED $(36.8 \%)$ for the prophylactic use; however, 
physicians likewise as often as possible chose phenobarbital (11.6\%) and valproic acid (7.8\%).

Table 1: Clinical/demographic characteristics of 119 glioma patients

\begin{tabular}{lc}
\hline Characteristics & $\mathbf{N}(\%)$ \\
& \\
\hline Gender & $73(61.34)$ \\
Male & $46(38.66)$ \\
Female & \\
Histology & $80(67.22)$ \\
Glioblastoma multiformae & $12(10.08)$ \\
Anaplastic oligodendroglioma & $10(8.4)$ \\
Anaplastic astrocytoma & $10(8.4)$ \\
Oligodendroglioma & $7(5.88)$ \\
Diffuse astrocytoma & \\
Location & $59(49.58)$ \\
Frontal & $31(26.05)$ \\
Temporal & $15(12.61)$ \\
Parietal & $9(7.56)$ \\
Basal ganglia & $7(5.88)$ \\
Occipital & $4(3.36)$ \\
Thalamus & \\
Therapy post-surgery & $18(15.13$ \\
Observation only & $101(84.87)$ \\
Irradiation and temozolomide &
\end{tabular}

Table 2: Seizure incidence in 119 glioma patients

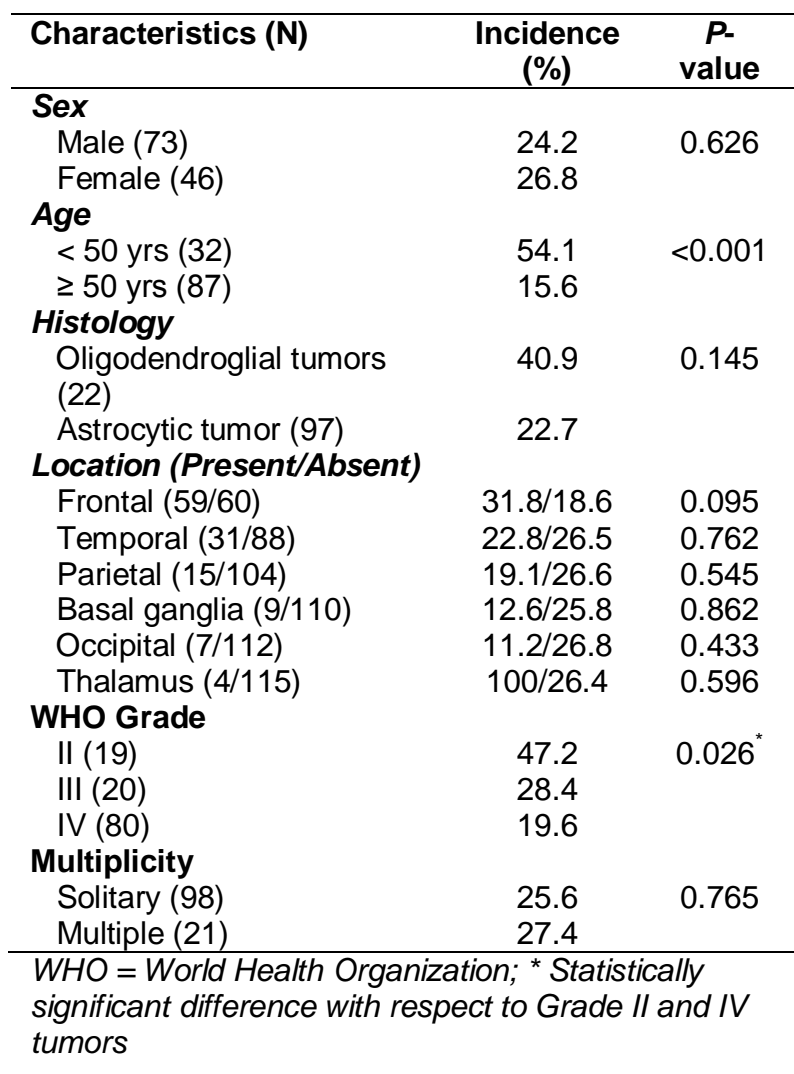

\section{Seizures amid early post-operative phase}

During surgery, all the patients were given AED prophylactically. Most of the patients received phenytoin $(85.7 \%)$. The rest were given
Phenobarbital (intramuscular injection/supposetory; $11.8 \%)$, oral valproic acid (1.6\%), and zonisamide $(0.8 \%)$. Ten patients (8.4\%) developed seizures amid initial postoperative week. Patients with seizure history prior to surgery experienced higher risk (17.2\%) than that of others significantly $(5.2 \%, p=0.045)$. The seizures happened more often during surgery day $(36.9 \%)$. The seizure incidence seen in phenobarbital-treated patients $(21.4 \%)$ was greater than patients on phenytoin $(6.9 \%)$; there was no statistically significant difference $(p=$ 0.266 ).

\section{Seizures amid follow up}

By the last follow up period, $73(61.3 \%)$ patients developed seizures. Seizures were seen most commonly in younger (<50 yrs, $84.1 \%)$ than older patients ( $\geq 50 \mathrm{yrs}, 52.6 \%, p<0.003)$. The main region with significant association with greater seizure incidence was frontal lobe $(p=$ 0.035 ). Overall seizure incidence and pertinent characteristics of patients are presented in Table 3.

Table 3: Overall seizure incidence in glioma patients

\begin{tabular}{|c|c|c|}
\hline Characteristics (N) & $\begin{array}{c}\text { Incidence } \\
(\%)\end{array}$ & $\begin{array}{c}P \text { - } \\
\text { value }\end{array}$ \\
\hline \multicolumn{3}{|l|}{ Sex } \\
\hline Male (73) & 64.2 & 0.846 \\
\hline Female (46) & 59.8 & \\
\hline \multicolumn{3}{|l|}{ Age } \\
\hline$<50$ yrs (32) & 84.1 & 0.002 \\
\hline$\geq 50$ yrs (87) & 52.6 & \\
\hline \multicolumn{3}{|l|}{ Histology } \\
\hline Oligodendroglial tumors (22) & 76.2 & 0.056 \\
\hline Astrocytic tumor (97) & 59.4 & \\
\hline \multicolumn{3}{|l|}{ Location (involved/not) } \\
\hline Frontal $(59 / 60)$ & $71.8 / 50.6$ & 0.035 \\
\hline Temporal (31/88) & $23.8 / 66.5$ & 0.056 \\
\hline Parietal $(15 / 104)$ & $50.1 / 62.6$ & 0.544 \\
\hline Basal ganglia $(9 / 110)$ & $24.6 / 63.8$ & 0.056 \\
\hline Occipital (7/112) & $33.2 .2 / 63.8$ & 0.083 \\
\hline Thalamus $(4 / 115)$ & $100 / 60.4$ & 0.296 \\
\hline \multicolumn{3}{|l|}{ WHO Grade } \\
\hline II (19) & 74.2 & $0.128^{\star}$ \\
\hline III (20) & 68.4 & \\
\hline IV $(80)$ & 56.6 & \\
\hline \multicolumn{3}{|l|}{ Multiplicity } \\
\hline Solitary (98) & 57.4 & 0.069 \\
\hline Multiple (21) & 77.2 & \\
\hline
\end{tabular}

Post-surgery, 12 patients didn't need any AED at any ailment phase; the rest of the 107 patients were given AED for seizure prophylaxis/control. Out of these patients, $34(28.6 \%)$ needed three/more AEDs for seizure control. Phenytoin was the most widely utilized AED $(n=60)$ 
postoperatively, followed by phenobarbital $(n=58)$ and valproic acid ( $n=56)$. Various patients likewise got carbamazepine $(n=14)$. At the point when seizures were not under control by those AEDs, levetiracetam $(n=20)$ or gabapentin $(n=$ 4 ) is included. Phenytoin usage lead to adverse effects customarily and seizure control failure often. Though valproic acid had lesser adverse effects, it poorly controlled seizures. Phenobarbital has substantial adverse event incidence, however controlled seizures superior to phenytoin and valproic acid. Carbamazepine showed good control of seizures, yet it was withdrawn often because of adverse effects. Skin rashes (phenytoin), dizziness (carbamazepine) and liver dysfunction (phenobarbital) were the common adverse effects. Interestingly, levetiracetam demonstrated good seizure control and was tolerated well, with withdrawn in one patient (somnolence).

Out of 80 patients with none of the preoperative seizures, 15 patients withdrew AED by the first post-operative week whereas the rest of 65 patients were given AED on a more extended period. Time to first seizure post-surgery as per prophylactic AED duration is depicted in Figure 1.

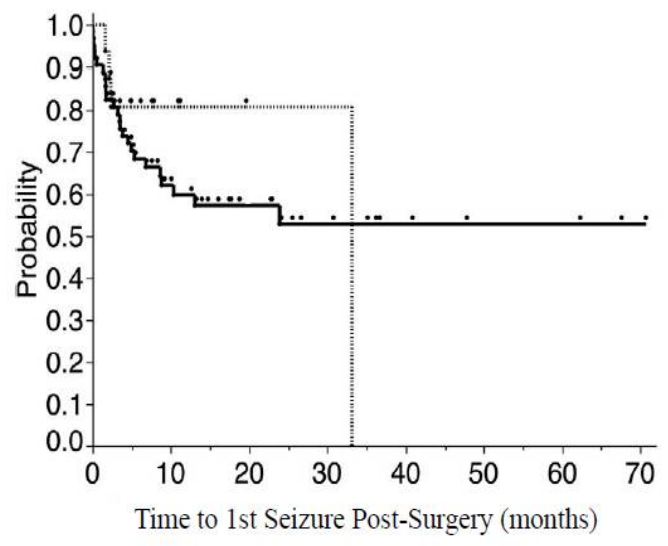

Figure 1: Time difference in first post-operative seizure with (dotted line) and without (solid line) prophylactic antiepileptic drugs beyond 1 week postsurgery in patients with no preoperative seizure history.

No significant time difference to first seizure postsurgery with/ without AED prophylaxis after 1 week post-surgery among patients with no preoperative seizure history was noted (log rank $p=0.584)$. AED continuation beyond first postoperative week granted no added benefit. Besides 15 patients, who discontinued AED, 39 additional patients discontinued later. Out of those 54 patients, 14 developed epileptic seizures within 6 months and again needed AED. The rest of 40 patients experienced no seizures and hence got none of the AEDs amid followup period (median, 7.2 months; range, $1.4-69.1$ months).

\section{DISCUSSION}

Primary brain tumours have greater seizure incidence than metastatic tumours [8]. In the present study, around $65 \%$ of the patients having supratentorial gliomas developed epilepsy. It was recently reported that glutamate released via glioma cells might be a potential cause of seizures in glioma patients [9]. Despite the fact that the exact way is unclear regarding epileptogenesis, tumours close to cortex and gradually progressive tumours accounted for higher seizure incidence [10]. In the present study, patients having oligodendroglia tumours (that habitually penetrate the cortex), had a high seizure incidence than that of astrocytic tumours. Also, the patients with the low grade tumours additionally demonstrated high seizure incidence. This information show that the patients who are relied upon for longer survival have a greater seizure risk. In this manner, seizure control is a central point in keeping up quality of life of patients having glioma.

Recently, researchers revealed an exceedingly low seizure incidence amid craniotomy without AEDs [11,12]. Lwu et al demonstrated $3 \%$ of patients experiencing seizures during perioperative period with none of the AEDs, while noting no seizures on AED prophylaxis [11]. This may be an underestimate of seizure incidence as they included only the patients having malignant glioma and no seizure history. Nonetheless, it is critical to take into account the need for AEDs in perioperative seizure prophylaxis from cost and benefit point of view. Contrary to the above report, in this study, $8.4 \%$ developed seizures in first week post craniotomy, despite the fact that all patients got prophylactic AEDs. Nearly $5.2 \%$ of patients with malignant tumours and no seizure history developed perioperative seizures. The greater perioperative seizure incidence in the patients on prophylactic AED compared to what was noted in Lwu et al study patients who were on no prophylaxis brings up issue of justified prophylactic utilization of AED in perioperative seizure control. Randomized trials are needed to elucidate if AEDs are fundamental in perioperative seizures prophylaxis in case of patients with no seizure history. On the other hand, there is supposedly a high seizure incidence amid awake craniotomy [13]. Besides, seizures amid awake surgery might interfere with neurological function monitoring [14]. Though fact that the seizure incidence might be low amid craniotomy, it is likely prophylactic AEDs are prescribed in surgeries of that type. 
In the present study, phenytoin was the widely utilized drug in perioperative seizure prophylaxis. Lately, researchers have demonstrated levetiracetam to be effective in seizure prophylaxis amid and not long after craniotomy [15]. Besides, levetiracetam is allegedly better than phenytoin for seizure prophylaxis during surgery [16].

In the event that seizures were very much in control amid surgery, we proceeded with the same AED in postoperative seizure prophylaxis in many patients. In this way, phenytoin was the most widely utilized AED postoperatively. However, phenytoin was very poorly tolerated and as a result, needed withdrawal within 2 months in greater than half of the patients. More regular reasons for withdrawal were adverse effects (rash, liver dysfunction) than unsatisfactory control of seizures.

Rash and dizziness were the most common reasons for carbamazepine withdrawal. Also, a few patients were on this agent due to poor acceptability. Levetiracetam acceptability was noted to be excellent with satisfactory seizure control and adverse events of lesser frequency. Although, significantly more number of psychiatric or behavioral adverse events were reported to be caused by levetiracetam than by gabapentin or lamotrigine [17]. Fortunately, none of the patients experienced the above kinds of adverse effects and in any case, it is important to be aware of such effects while utilizing levetiracetam for seizure control.

In spite of the fact of secure withdrawal of AEDs in 39/119 patients (32.8\%), more than two AEDs were needed for seizure control in 34/119 patients $(28.6 \%)$. These outcomes show how troublesome the seizure control can be in glioma patients. Additionally, during treatment, it is critical to watch out for interactions amongst AEDs and different drugs utilized for brain tumour treatment. The majority of the already accessible AEDs are metabolized by means of the cytochrome P450 pathway. Some AEDs influence the action of that enzyme. For instance, valproic acid inhibits CYP 2C19 whereas phenytoin and phenobarbital induces CYP 3A4 [18]. Then again, a significant number of the agents that are often used for brain tumour treatment are additionally metabolized via this pathway and might likewise influence it. CYP 3A4 induction by dexamethasone [19] and its reduction by cimetidine [20] are well known. Also, valproic acid inhibits glucuronidation while carbamazepine, phenytoin and phenobarbital induce it. Unstable AED concentrations due to such interactions leads to unsatisfactory seizure control. It is vital to consider adverse event risk or activity reduction of anti-cancer drugs due to metabolic interactions amongst AEDs and anticancer agents. Levetiracetam and gabapentin are not metabolized and are generally discharged by the kidneys. Hence they do not have much interaction with other therapeutic agents and are therefore safer to use with other anticancer drugs.

Klein et al earlier revealed that patients having glioma demonstrate poor cognitive function when compared to healthy controls. It was also described that treatment with AEDs, as opposed to seizure recurrence, negatively influences cognitive function [21]. To prevent AED adverse effects, along with deterioration of cognition, the American Academy of Neurology Quality Standards Subcommittee has developed guidelines which prescribe tapering and discontinuation of AED beyond the first postoperative week in the patients having no seizure history [22]. In the present study, AED continuation beyond 1 week post craniotomy in the patients with no seizure history did not prevent postoperative seizure onset. In this way, our outcomes reinforce the above mentioned guidelines in regards to AED prophylaxis for the patients with no prior seizures. Nevertheless, it is crucial to take account that no seizures prior to craniotomy does not ensure no seizure course post-surgery. In the present study, greater than $40 \%$ of the patients with no seizure prior to surgery experienced the seizures post-surgery, despite on AEDs.

Epilepsy in brain tumour patients is a distinctive form where the seizure risk might change as per tumour status. Recurrence of tumour and alterations in encompassing brain tissue due to therapy, for example, necrosis post radiation treatment, might change the seizure risk. Hence, medication discontinuation should be examined carefully, despite the fact that these drugs can lead to decreased cognitive function. Newer AEDs, namely levetiracetam, gabapentin and lamotrigine on the other hand, are apparently safe with respect to the cognitive function [23]. Levetiracetam, apparently enhances memories in the animal models [24] and enhances quality of life in brain tumour patients having epilepsy [25]. Of late, verbal memory improvement in highgrade glioma patients on levetiracetam has been demonstrated [26]. We prescribe picking this AED in patients where AED discontinuation is not advisable due to unsteady conditions of tumour. 


\section{Limitations of the study}

Certain innate limitations need consideration during results interpretation of of present study. This single-center study has limited number of patients, hence the results generalization should be done with great care. We were not able to assess all variables and were limited by the treating physicians in regards to completeness of data or proper documentation. Limited data was captured.

\section{CONCLUSION}

Effective prophylaxis of seizure and acceptable adverse event profile should be considered significant in AEDs selection. Additionally, in brain tumour patients suffering epilepsy, it is important to consider drug interactions with other therapeutic agents and cognitive impairment risk. The lower cognitive impairment risk and the renal discharge associated with newer AEDs make them more suitable than the older therapeutic agents for epilepsy control in brain tumour patients.

\section{DECLARATIONS}

\section{Conflict of Interest}

No conflict of interest associated with this work.

\section{Contribution of Authors}

The authors declare that this work was done by the authors named in this article and all liabilities pertaining to claims relating to the content of this article will be borne by them.

\section{Open Access}

This is an Open Access article that uses a funding model which does not charge readers or their institutions for access and distributed under the terms of the Creative Commons Attribution License (http://creativecommons.org/licenses/by 14.0) and the Budapest Open Access Initiative (http://www.budapestopenaccessinitiative.org/rea d), which permit unrestricted use, distribution, and reproduction in any medium, provided the original work is properly credited.

\section{REFERENCES}

1. Taphoorn MJ. Neurocognitive sequelae in the treatment of low grade gliomas. Semin Oncol 2003; 30: 45-48.
2. van Breemen MS, Wilms EB, Vecht CJ. Epilepsy in patients with brain tumours: epidemiology, mechanisms, and management. Lancet Neurol 2007; 6: 421-430.

3. Rudà R, Trevisan E, Soffietti R. Epilepsy and brain tumours. Curr Opin Oncol 2010; 22: 611-620.

4. Herman ST. Epilepsy after brain insult: targeting epileptogenesis. Neurology 2002; 59 (suppl 5): S21S26.

5. Olafsson E, Ludvigsson P, Gudmundsson G, Hesdorffer $D$, Kjartansson $O$, Hauser WA. Incidence of unprovoked seizures and epilepsy in Iceland and assessment of the epilepsy syndrome classifi cation: a prospective study. Lancet Neurol 2005; 4: 627-634.

6. Hauser WA, Annegers JF, Kurland LT. Incidence of epilepsy and unprovoked seizures in Rochester, Minnesota: 1935-1984. Epilepsia 1993; 34: 453-468.

7. Stupp R, Hegi ME, Mason WP, van den Bent MJ, Taphoorn MJ, Janzer RC, Ludwin SK, Allgeier A, Fisher $B$, Belanger $K$, et al. Effects of radiotherapy with concomitant and adjuvant temozolomide versus radiotherapy alone on survival in glioblastoma in a randomised phase III study: 5-year analysis of the EORTC-NCIC trial. Lancet Oncol 2009; 10(5): 459-466.

8. Oberndorfer S, Schmal T, Lahrmann H, Urbanits $S$, Lindner $K$, Grisold $W$. The frequency of seizures in patients with primary brain tumours or cerebral metastases. An evaluation from the Ludwig Boltzmann Institute of Neuro-Oncology and the Department of Neurology, Kaiser Franz Josef Hospital, Vienna. Wien Klin Wochenschr 2002; 114(21-22): 911-916.

9. Buckingham SC, Campbell SL, Haas BR, Montana V, Robel S, Ogunrinu T, Sontheimer H. Glutamate release by primary brain tumours induces epileptic activity. Nat Med 2011; 17(10): 1269-1274.

10. van Breemen MS, Wilms EB, Vecht CJ. Epilepsy in patients with brain tumours: epidemiology, mechanisms, and management. Lancet Neurol 2007; 6: 421-430.

11. Lwu S, Hamilton MG, Forsyth PA, Cairncross JG, Parney IF. Use of peri-operative anti-epileptic drugs in patients with newly diagnosed high grade malignant glioma: a single center experience. J Neurooncol 2010; 96(3): 403-408.

12. Sughrue ME, Rutkowski MJ, Chang EF, Shangari G, Kane AJ, McDermott MW, Berger MS, Parsa AT. Postoperative seizures following the resection of convexity meningiomas: are prophylactic anticonvulsants indicated? Clinical article. J Neurosurg 2011; 114(3): 705-709.

13. Serletis $D$, Bernstein $M$. Prospective study of awake craniotomy used routinely and nonselectively for supratentorial tumours. J Neurosurg 2007; 107(1): 1-6.

14. Nossek E, Matot I, Shahar T, Barzilai O, Rapoport $Y$, Gonen $T$, Sela G, Korn A, Hayat D, Ram Z. Failed awake craniotomy: a retrospective analysis in 424 patients undergoing craniotomy for brain tumour. $J$ Neurosurg 2013; 118(2): 243-249.

15. Bähr O, Hermission M, Rona S, Rieger J, Nussbaum S, Körtvelyessy $P$, Franz K, Tatagiba M, Seifert V, Weller 
$M$, Steinbach JP. Intravenous and oral levetiracetam in patients with a suspected primary brain tumour and symptomatic seizures undergoing neurosurgery: the HELLO trial. Acta Neurochir (Wien) 2012; 154(2): 229235.

16. Kern K, Schebesch KM, Schlaier J, Hansen E, Feigl GC, Brawanski AT, Lange M. Levetiracetam compared to phenytoin for the prevention of postoperative seizures after craniotomy for intracranial tumours in patients without epilepsy. J Clin Neurosci 2012; 19(1): 99-100.

17. Weintraub D, Buchsbaum R, Resor SR Jr., Hirsch LJ. Psychiatric and behavioral side effects of the newer antiepileptic drugs in adults with epilepsy. Epilepsy Behav 2007; 10(1): 105-110.

18. Vecht CJ, Wagner GL, Wilms EB. Interactions between antiepileptic and chemotherapeutic drugs. Lancet Neurol 2003; 2(7): 404-409.

19. Teo YL, Saetaew M, Chanthawong S, Yap YS, Chan EC, Ho HK, Chan A. Effect of CYP3A4 inducer dexamethasone on hepatotoxicity of lapatinib: clinical and in vitro evidence. Breast Cancer Res Treat 2012; 133(2): 703-711.

20. Akiyoshi T, Saito T, Murase S, Miyazaki M, Murayama N, Yamazaki H, Guengerich FP, Nakamura K, Yamamoto $K$, Ohtani $H$. Comparison of the inhibitory profiles of itraconazole and cimetidine in cytochrome P450 3A4 genetic variants. Drug Metab Dispos 2011; 39(4): 724728.
21. Klein M, Engelberts NHJ, van der Ploeg HM, KasteleijnNolst Trenité DG, Aaronson NK, Taphoorn MJ, Baaijen $H$, Vandertop WP, Muller M, Postma TJ, et al. Epilepsy in low-grade gliomas: the impact on cognitive function and quality of life. Ann Neurol 2003; 54: 514-520.

22. Grants $M J$, Cole BF, Forsyth $P A$, Recht $L D$, Wen $P Y$, Chamberlain MC, Grossman SA, Cairncross JG. Practice parameter: Anticonvulsant prophylaxis in patients with newly diagnosed brain tumours. Report of the Quality Standards Subcommittee of the American Academy of Neurology. Neurol 2000; 54(10): 18861893.

23. Eddy CM, Rickards HE, Cavanna AE. The cognitive impact of antiepileptic drugs. Ther Adv Neurol Disord 2011; 4(6): 385-407.

24. Celikyurt IK, Ulak G, Mutlu O, Akar FY, Mulayim S, Erden F, Komsuoglu SS. Positive impact of levetiracetam on emotional learning and memory in naive mice. Life Sci 2012; 90(5-6): 185-189.

25. Maschio M, Dinapoli L, Sperati F, Pace A, Fabi A, Vidiri $A$, Muti $P$. Levetiracetam monotherapy in patients with brain tumour-related epilepsy: seizure control, safety, and quality of life. J Neurooncol 2011; 104(1): 205-214.

26. de Groot M, Douw L, Sizoo EM, Bosma I, Froklage FE, Heimans JJ, Postma TJ, Klein M, Reijneveld JC. Levetiracetam improves verbal memory in high-grade glioma patients. Neuro Oncol 2013; 15(2): 216-223. 